\title{
Electromagnetic perturbations of black holes in general relativity coupled to nonlinear electrodynamics
}

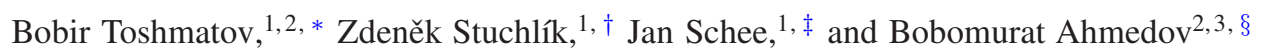 \\ ${ }^{1}$ Institute of Physics and Research Centre of Theoretical Physics and Astrophysics, \\ Faculty of Philosophy \& Science, Silesian University in Opava, \\ Bezručovo náměstí 13, CZ-74601 Opava, Czech Republic \\ ${ }^{2}$ Ulugh Beg Astronomical Institute, Astronomicheskaya 33, Tashkent 100052, Uzbekistan \\ ${ }^{3}$ National University of Uzbekistan, Tashkent 100174, Uzbekistan
}

\begin{abstract}
The electromagnetic (EM) perturbations of the black hole solutions in general relativity coupled to nonlinear electrodynamics (NED) are studied for both electrically and magnetically charged black holes, assuming that the EM perturbations do not alter the spacetime geometry. It is shown that the effective potentials of the electrically and magnetically charged black holes related to test perturbative NED EM fields are related to the effective metric governing the photon motion, contrary to the effective potential of the linear electrodynamic (Maxwell) field that is related to the spacetime metric. Consequently, corresponding quasinormal (QN) frequencies differ as well. As a special case, we study new family of the NED black hole solutions which tend in the weak field limit to the Maxwell field, giving the Reissner-Nordström (RN) black hole solution. We compare the NED Maxwellian black hole QN spectra with the RN black hole QN spectra.
\end{abstract}

\section{INTRODUCTION}

It is well known fact that most of the exact solutions of Einstein's equations have curvature singularity which is still one of the unexplained problems of the general relativity. In order to escape from this inexplicable property of the spacetime, obtaining the black hole solutions without singularity, i.e., the regular black hole solutions, has been urged on. One of the simplest ways to obtain a regular black hole solution is coupling general relativity to some other fundamental fields such as those described by the NED. One of the attractive properties of the NED is the ability to eliminate the curvature singularity from the black hole solutions [1]

It is known that observations [7] and analysis of data [8] show that the real astrophysical black holes are rotating. However, constructing the rotating black hole solution in general relativity coupled to NED is another challenge that has not been solved completely. So far, several authors have made a lot of efforts to take rotating black hole solutions from existing spherically symmetric ones by using the Newman-Janis algorithm [9] 13] and Gürses-Gürsey algorithm [14, 15]. Unfortunately, these rotating solutions are not always representing exact solutions of the whole set of field equations of the theory. Namely, the energy-momentum tensor of the rotating regular black hole solutions obtained by the Newman-Janis algorithm sometimes does not correspond exactly to the NED equations [16] and such rotating solutions could be considered only as approximative solutions. Moreover, induced rotation parameter violates the weak energy condition of these approximate solutions [10, 11, 17].

One of the special properties of the NED field is that in such a field photon does not follow the null geodesics of the

\footnotetext{
bobir.toshmatov@fpf.slu.cz

zdenek.stuchlik@fpf.slu.ct

$\ddagger$ ian.schee@fpf.slu.cy

$\S$ ahmedov@ astrin.uz
}

background spacetime metric anymore, instead, it propagates along the null geodesics of an effective metric which is characterized by the non-linearities of the field [18.23.

In the present paper we focus our attention to the behavior of the dynamical response of the spherically symmetric, magnetically and electrically charged black holes representing exact solutions of coupled Einstein's gravity and NED to small EM perturbations. Especially, we are going to determine if it is possible to distinguish the black holes related to the NED from the black holes related to the standard linear electrodynamics due to their response to EM perturbations.

Perturbations of black holes imply the study of stability of their spacetime. The stability of the various black holes in NED has been studied in 24 26]. Since the system is open, if the black hole is stable against small perturbations, it relaxes to its equilibrium state by losing energy through emitting gravitational, EM or scalar radiation, depending on the underlying perturbations. The most important part of this radiation is an intermediate one which is called ringdown phase that is characterized by a (complex) frequency. Its real and imaginary parts represent frequency of real oscillations and their damping rate, respectively. So far, different types of perturbations of the various regular black holes in NED have been studied in 2733 .

The paper is organized as follows. In Sec. II we present the formalism to construct the electrically and magnetically charged black hole solutions in GR coupled to the NED. In Sec. III we present new family of the magnetically charged black hole solutions. Axial EM perturbations of the electrically and magnetically charged spherically symmetric NED black holes and the master equation for them are presented in Sec. IV. QN frequencies, temporal evolution and stability of EM perturbations of the new obtained NED black hole solution are studied in Sec.V. In Sec.VI, we study and make comparison of the QN frequencies of the NED black holes and the standard linear electrodynamics RN black holes in the eikonal (large multipole number or high frequency) regime. Finally, we present conclusions implied by our results in Sec. VII. In this paper we mainly use geometrized units $c=1=G$. Fur- 
thermore, we adopt $(-,+,+,+)$ convention for the signature of the metric.

\section{GR COUPLED TO NED}

The action of Einstein's gravity (GR) coupled to the NED is given as

$$
S=\frac{1}{16 \pi} \int d^{4} x \sqrt{-g}(R-\mathscr{L})
$$

where $g$ is the determinant of the metric tensor $g_{\mu \nu}, R$ is the scalar curvature, and $\mathscr{L}$ represents the Lagrangian density of the NED field that is function of the electrodynamic field strength, $\mathscr{L}=\mathscr{L}(F)$, with $F=F_{\mu \nu} F^{\mu \nu}$, where $F_{\mu \nu}$ is the electrodynamic field tensor that can be written in terms of a gauge potential as $F_{\mu \nu}=\partial_{\mu} A_{\nu}-\partial_{\nu} A_{\mu}$. Definition of the EM field tensor shows that $F_{\mu \nu}$ is anti-symmetric and it has only six independent components.

By neglecting the EM sources, one can write the covariant equations of motion in the form

$$
\begin{aligned}
& G_{\mu \nu}=T_{\mu \nu}, \\
& \nabla_{\nu}\left(\mathscr{L}_{F} F^{\mu \nu}\right)=0,
\end{aligned}
$$

where the Einstein tensor $G_{\mu \nu}=R_{\mu \nu}-R g_{\mu \nu} / 2$ and $T_{\mu \nu}$ is the energy-momentum tensor of the EM field, determined by the relation

$$
T_{\mu \nu}=2\left(\mathscr{L}_{F} F_{\mu}^{\alpha} F_{\nu \alpha}-\frac{1}{4} g_{\mu \nu} \mathscr{L}\right),
$$

with $\mathscr{L}_{F}=\partial_{F} \mathscr{L}$.

The line element of the static spherically symmetric black hole reads

$$
d s^{2}=-f(r) d t^{2}+\frac{d r^{2}}{f(r)}+r^{2}\left(d \theta^{2}+\sin ^{2} \theta d \phi^{2}\right),
$$

where the lapse function $f(r)$ depends on the NED field. This line element satisfies the symmetry $G_{t}^{t}=G_{r}^{r}$. The ansatz for the EM field can be written in general form as

$$
\bar{A}_{\mu}=\varphi(r) \delta_{\mu}^{t}-Q_{m} \cos \theta \delta_{\mu}^{\phi},
$$

where $\varphi(r)$ is electric potential, while $Q_{m}$ is the magnetic charge. Below we construct electrically and magnetically charged black hole solutions in general relativity coupled to the NED by the method of Bronnikov [3].

\section{A. Electrically charged black hole solution}

The ansatz of the electrically charged black hole solution is given as $\bar{A}_{t}=\varphi(r)$. Then, the EM field tensor has only nonzero component $F_{t r}=-F_{r t}=-\varphi^{\prime}(r)$. By the relation $F=F_{\mu \nu} F^{\mu \nu}$ we obtain the EM field strength as $F=-2 \varphi^{\prime 2}$. Let us consider the metric function $f(r)$ given in the form

$$
f(r)=1-\frac{2 m(r)}{r} .
$$

Then from the Einstein equation (2), we obtain only two independent equations

$$
\begin{aligned}
& r^{2}\left(\mathscr{L}+4 \mathscr{L}_{F} \varphi^{\prime 2}\right)-4 m^{\prime}=0, \\
& \mathscr{L} r-2 m^{\prime \prime}=0,
\end{aligned}
$$

By solving above given equations we obtain

$$
\begin{aligned}
& \mathscr{L}=\frac{2 m^{\prime \prime}}{r}, \\
& \mathscr{L}_{F}=\frac{2 m^{\prime}-r m^{\prime \prime}}{2 r^{2} \varphi^{\prime 2}},
\end{aligned}
$$

One can see from Eqs. 110) and (11) that if the mass function does not depend on radius, $m(r)=M$, the Lagrangian density of the electrodynamic field vanishes, and we arrive at the solution of the general relativity itself, i.e. the Schwarzschild metric. From equations of motion ( $\beta$ ), the total electric charge inside the sphere with radius $r$ reads

$$
Q_{e}=r^{2} \mathscr{L}_{F} \varphi^{\prime} .
$$

By substituting (11) to (12) and solving the differential equation, one obtains the electric potential $\varphi(r)$ in the form

$$
\varphi=\frac{3 m-r m^{\prime}}{2 Q_{e}}+C,
$$

where $C$ is an integration constant. If we take the linear electrodynamic field, i.e., the Maxwell field, our solution reduces to the RN black hole spacetime with $m(r)=M+Q_{e}^{2} / 2 r$. Then, the electric potential (13) takes the form $\varphi=Q_{e} / r$.

By choosing the mass function related to the electric field one can construct singular and regular black hole solutions in NED.

\section{B. Magnetically charged black hole solution}

The ansatz of the magnetically charged spherically symmetric black hole spacetime is given by $\bar{A}_{\phi}=-Q_{m} \cos \theta$. Nonzero components of the EM field tensor are $F_{\theta \phi}=$ $Q_{m} \sin \theta=-F_{\phi \theta}$. EM field strength is $F=2 Q_{m}^{2} / r^{4}$. Solving the Einstein equations (2) for this case we obtain two independent equations

$$
\begin{aligned}
& \mathscr{L} r^{2}-4 m^{\prime}=0, \\
& 4 \mathscr{L}_{F} q^{2}-\mathscr{L} r^{4}+2 r^{3} m^{\prime \prime}=0,
\end{aligned}
$$

By solving Eqs. (14) and (15) we obtain

$$
\begin{aligned}
& \mathscr{L}=\frac{4 m^{\prime}}{r^{2}}, \\
& \mathscr{L}_{F}=\frac{r^{2}\left(2 m^{\prime}-r m^{\prime \prime}\right)}{2 Q_{m}^{2}},
\end{aligned}
$$

\footnotetext{
${ }^{1}$ In $[$ it has been shown that in order for the solution to represent the regular black hole spacetime, one must choose the mass function so that it satisfies conditions: $\lim _{r \rightarrow 0} m / r^{3}=$ finite, $\lim _{r \rightarrow 0} m^{\prime} / r^{2}=$ finite, $\lim _{r \rightarrow 0} m^{\prime \prime} / r=$ finite.
} 
If we assume that the EM field is linear, i.e., the Maxwell field, $\mathscr{L}=F$ and $\mathscr{L}_{F}=1$, then, by solving the above equations we arrive at the mass function $m=M-Q_{m}^{2} / 2 r$ that represents again the RN black hole spacetime which is the solution of the Einstein-Maxwell equations.

\section{NEW MAGNETICALLY CHARGED BLACK HOLE SOLUTION}

In the paper [5], the authors proposed the formalism presented in section II and obtained some black hole solutions in GR coupled to NED. They generalized the solutions by choosing the Lagrangian density in the form

$$
\mathscr{L}=\frac{4 \mu}{\alpha} \frac{(\alpha F)^{\frac{\nu+3}{4}}}{\left[1+(\alpha F)^{\frac{\nu}{4}}\right]^{1+\frac{\mu}{\nu}}},
$$

where $\mu>0$ is a dimensionless constant which characterizes the strength of nonlinearity of the electrodynamic field, and $\alpha>0$ is constant parameter which is in the unit of length squared; $\alpha$ is introduced into theory by the definition $Q_{m}=q^{2} / \sqrt{2 \alpha}$. For the magnetically charged nonlinear electrodynamic field they obtained the solution in the following form [5]:

$$
f(r)=1-\frac{2 M}{r}-\frac{2 q^{3}}{\alpha} \frac{r^{\mu-1}}{\left(r^{\nu}+q^{\nu}\right)^{\frac{\mu}{\nu}}}
$$

where $q$ is magnetic charge parameter and $\nu>0$ is dimensionless constant. $M$ is the pure gravitational mass, let us say Schwarzschild mass.

However, our calculations show that the Lagrangian density (18) gives more general solution in the form

$$
f(r)=1-\frac{2 M}{r}+\frac{2 q^{3}}{\alpha r}-\frac{2 q^{3}}{\alpha} \frac{r^{\mu-1}}{\left(r^{\nu}+q^{\nu}\right)^{\frac{\mu}{\nu}}}
$$

Comparing Eqs. (19) and 20), one can easily notice that the difference in the mass functions is the ratio $-q^{3} / \alpha$ which cannot be dropped. Mathematically, dropping it also satisfies all equations, however, dropping of this term is equivalent to $q=0$, which eliminates the last ratio as well. The asymptotic behaviour of $(20)$ gives the Arnowitt-Deser-Misner (ADM) mass of the black hole to be $M_{A D M}=M$.

As shown in [5], the black hole solution (\$) with the metric function 19 is singular at origin, $r=0$, and regular only if the pure gravitational mass is neglected, $M=0$, and $\mu \geq 3$. However, the black hole solution with metric function 20 is singular at $r=0$, even if $M=0$. The only way to make it regular everywhere in the spacetime is to assume that the gravitational mass is equal to

$$
M=\frac{q^{3}}{\alpha},
$$

\footnotetext{
${ }^{2}$ In the paper [D] the ADM mass is given as $M_{A D M}=M+q^{3} / \alpha$.
}

with $\mu \geq 3$. Then one can write the metric function $(20)$ in the following form:

$$
f(r)=1-\frac{2 M r^{\mu-1}}{\left(r^{\nu}+q^{\nu}\right)^{\frac{\mu}{\nu}}}
$$

Here $M$ is still pure gravitational mass 3. One may argue that considering the gravitational mass is constant and playing freely with value of the charge parameter is impossible, since they are related to each other due to (21). However, fortunately, we have one more free parameter $\alpha$ which can provide the gravitational mass to be constant even if the value of charge changes.

\section{AXIAL EM PERTURBATIONS OF NED BLACK HOLES}

In this section we study axial EM perturbations of black holes in NED by introducing the axial perturbations into gauge potential (6) as

$$
A_{\mu}=\bar{A}_{\mu}+\delta A_{\mu}
$$

considering the perturbations given in the form

$$
\delta A_{\mu}=\sum_{\ell, m}\left(\left[\begin{array}{c}
0 \\
0 \\
\Psi^{\ell m}(t, r) \partial_{\phi} Y_{\ell m}(\theta, \phi) / \sin \theta \\
-\Psi^{\ell m}(t, r) \sin \theta \partial_{\theta} Y_{\ell m}(\theta, \phi)
\end{array}\right]\right)
$$

where $Y_{\ell m}(\theta, \phi)$ is the spherical harmonic function of degree $\ell$ and order $m$, f related to the angular coordinates $\theta$ and $\phi$. Below we study electrically and magnetically charged black hole cases separately.

\section{A. Electrically charged black hole}

The gauge potential for the electrically charged spherically symmetric black hole solution is given in the general form $\bar{A}_{\mu}=\varphi(r) \delta_{\mu}^{t}$.

The nonvanishing covariant components of the EM field tensor of the 4-potential (23) with perturbation (24) are given by

$$
\begin{aligned}
F_{t r} & =-\partial_{r} \varphi \\
F_{t \theta} & =\frac{1}{\sin \theta} \partial_{t} \Psi^{\ell m} \partial_{\phi} Y_{\ell m}, \\
F_{t \phi} & =-\sin \theta \partial_{t} \Psi^{\ell m} \partial_{\theta} Y_{\ell m}, \\
F_{r \theta} & =\frac{1}{\sin \theta} \partial_{r} \Psi^{\ell m} \partial_{\phi} Y_{\ell m}, \\
F_{r \phi} & =-\sin \theta \partial_{r} \Psi^{\ell m} \partial_{\theta} Y_{\ell m}, \\
F_{\theta \phi} & =-\Psi^{\ell m}\left[\partial_{\theta}\left(\sin \theta \partial_{\theta} Y_{\ell m}\right)+\frac{1}{\sin \theta} \partial_{\phi}^{2} Y_{\ell m}\right] \\
& =-\ell(\ell+1) \Psi^{\ell m} \sin \theta Y_{\ell m} .
\end{aligned}
$$

\footnotetext{
${ }^{3}$ In the paper [f] the regular solution also takes the form of 22, but $M$ is the electromagnetically induced mass.

${ }^{4}$ For the EM perturbations $\ell=1,2,3, \ldots$ and $m= \pm 1,2, \pm 3, \ldots, \pm \ell$.
} 
From the relation $F^{\mu \nu}=g^{\mu \alpha} g^{\nu \beta} F_{\alpha \beta}$, we find the nonvanishing contravariant components of the EM field tensor

$$
\begin{aligned}
F^{t r} & =\partial_{r} \varphi \\
F^{t \theta} & =-\frac{1}{f r^{2} \sin \theta} \partial_{t} \Psi^{\ell m} \partial_{\phi} Y_{\ell m}, \\
F^{t \phi} & =\frac{1}{f r^{2} \sin \theta} \partial_{t} \Psi^{\ell m} \partial_{\theta} Y_{\ell m}, \\
F^{r \theta} & =\frac{f}{r^{2} \sin \theta} \partial_{r} \Psi^{\ell m} \partial_{\phi} Y_{\ell m}, \\
F^{r \phi} & =-\frac{f}{r^{2} \sin \theta} \partial_{r} \Psi^{\ell m} \partial_{\theta} Y_{\ell m}, \\
F^{\theta \phi} & =-\frac{\ell(\ell+1)}{r^{4} \sin \theta} \Psi^{\ell m} Y_{\ell m} .
\end{aligned}
$$

By combining (25) and (26) and taking only first order perturbations, we find the EM field strength $F$ in the form

$$
F \approx-2 \varphi^{\prime 2} \text {. }
$$

Hereafter, prime denotes the partial derivative with respect to $r\left(X^{\prime}=\partial_{r} X\right)$. One can see from (27) that in the perturbation of gauge potential, EM field strength, $\bar{F}$, remains unchanged as

$$
\mathscr{L}_{F}=\overline{\mathscr{L}}_{\bar{F}} .
$$

By inserting (26) into (3) we get the relation.

$$
\begin{aligned}
\partial_{t} F^{\mu t}+ & \frac{1}{r^{2} \mathscr{L}_{F}} \partial_{r}\left(r^{2} \mathscr{L}_{F} F^{\mu r}\right)+\frac{1}{\sin \theta} \partial_{\theta}\left(\sin \theta F^{\mu \theta}\right) \\
& +\partial_{\phi} F^{\mu \phi}=0,
\end{aligned}
$$

For $\mu=t$, we arrive at Gauss's law

$$
\varphi=\int \frac{Q_{e}}{r^{2} \mathscr{L}_{F}} d r
$$

where $Q_{e}$ is total charge inside the sphere with radius $r$. For the RN black hole case $\mathscr{L}_{F}=1$, therefore, $\varphi_{R N}=-Q_{e} / r$ justifies above relation.

For the case of $\mu=r$, equation (3) has infinite solutions. Finally, for $\mu=\theta$ and $\mu=\phi$ we arrive at the same equation

$$
-\frac{\partial^{2} \Psi}{\partial t^{2}}+\frac{f}{\mathscr{L}_{F}}\left(f \mathscr{L}_{F} \Psi^{\prime}\right)^{\prime}+f \frac{\ell(\ell+1)}{r^{2}} \Psi=0 .
$$

For simplicity, we choose the function $\Psi$ in the form

$$
\Psi=\frac{1}{\sqrt{\mathscr{L}_{F}}} \Phi
$$

and introducing the new radial, so-called tortoise coordinate $d x=d r / f$, we rewrite the equation (31) in terms of the new wave function and arrive at the well-known Schrödinger-like wave equation

$$
\left[-\frac{\partial^{2}}{\partial t^{2}}+\frac{\partial^{2}}{\partial x^{2}}-V_{e}(r)\right] \Phi_{e}(r, t)=0,
$$

where the effective potential is given by

$$
V_{e}(r)=f\left[\frac{\ell(\ell+1)}{r^{2}}-\frac{f \mathscr{L}_{F}^{\prime 2}-2 \mathscr{L}_{F}\left(f \mathscr{L}_{F}^{\prime}\right)^{\prime}}{4 \mathscr{L}_{F}^{2}}\right]
$$

where $\mathscr{L}_{F}$ is given by the expression (11). As it has already been pointed out that $F$ and $\mathscr{L}_{F}$ depend explicitly and implicitly only on $r$, respectively. Therefore, one can write the first and second order radial derivatives of $\mathscr{L}_{F}$ as $\mathscr{L}_{F}^{\prime}=\mathscr{L}_{F F} / F^{\prime}$ and $\mathscr{L}_{F}^{\prime \prime}=\left(\mathscr{L}_{F F F}-\mathscr{L}_{F F} F^{\prime \prime}\right) / F^{\prime 2}$, respectively. However, when the black hole solution is constructed by the means shown in section II, one will have a problem on expressing the Lagrangian density $\mathscr{L}$ explicitly as a function of the EM field strength $F$. Therefore, in this case it is better to keep the Lagrangian density $\mathscr{L}$ as a function of $r$ as in (10) and (11). Moreover, here $\mathscr{L}_{F F}=\mathscr{L}_{F}^{\prime} / F^{\prime}$. For the RN black hole $\mathscr{L}_{F}=1$ or $m=M-Q_{e}^{2} / 2 r\left(f=1-2 M / r+Q_{e}^{2} / r^{2}\right)$, and for the other black holes which are not solution of electrodynamics $(F=0)$, we obtain the well-known potential

$$
V(r)=f \frac{\ell(\ell+1)}{r^{2}} .
$$

\section{B. Magnetically charged black hole}

The ansatz of the black hole with magnetic charge reads $\bar{A}_{\mu}=-Q_{m} \cos \theta \delta_{\mu}^{\phi}$. Again we add perturbations (24) to the 4-potential as (23) and write the nonzero covariant components of the EM field tensor

$$
\begin{aligned}
& F_{t \theta}=\frac{1}{\sin \theta} \partial_{t} \Psi^{\ell m} \partial_{\phi} Y_{\ell m}, \\
& F_{t \phi}=-\sin \theta \partial_{t} \Psi^{\ell m} \partial_{\theta} Y_{\ell m}, \\
& F_{r \theta}=\frac{1}{\sin \theta} \partial_{r} \Psi^{\ell m} \partial_{\phi} Y_{\ell m}, \\
& F_{r \phi}=-\sin \theta \partial_{r} \Psi^{\ell m} \partial_{\theta} Y_{\ell m}, \\
& F_{\theta \phi}=\sin \theta\left(Q_{m}-\ell(\ell+1) \Psi^{\ell m} Y_{\ell m}\right) .
\end{aligned}
$$

By the relation $F^{\mu \nu}=g^{\mu \alpha} g^{\nu \beta} F_{\alpha \beta}$ the nonzero contravariant components of the EM field tensor can be written as

$$
\begin{aligned}
F^{t \theta} & =-\frac{1}{f r^{2} \sin \theta} \partial_{t} \Psi^{\ell m} \partial_{\phi} Y_{\ell m}, \\
F^{t \phi} & =\frac{1}{f r^{2} \sin \theta} \partial_{t} \Psi^{\ell m} \partial_{\theta} Y_{\ell m}, \\
F^{r \theta} & =\frac{f}{r^{2} \sin \theta} \partial_{r} \Psi^{\ell m} \partial_{\phi} Y_{\ell m}, \\
F^{r \phi} & =-\frac{f}{r^{2} \sin \theta} \partial_{r} \Psi^{\ell m} \partial_{\theta} Y_{\ell m}, \\
F^{\theta \phi} & =\frac{1}{r^{4} \sin \theta}\left(Q_{m}-\ell(\ell+1) \Psi^{\ell m} Y_{\ell m}\right) .
\end{aligned}
$$

The EM field strength $F$ up to the first order perturbation terms

$$
F \approx \frac{2 Q_{m}^{2}}{r^{4}}-\frac{4 Q_{m} \ell(\ell+1) \Psi^{\ell m} Y_{\ell m}}{r^{4}} .
$$

One can see from (38) that unlike the case of the electrically charged black hole, axial perturbations change the EM field strength. In (38), the first term corresponds to the unperturbed EM field strength, $\bar{F}$, while the second term is the contribution of the perturbation to the field strength, $\delta F$, i.e., $F=\bar{F}+\delta F$. 
Because of the change in the argument $\bar{F}$, the expression of $\overline{\mathscr{L}}_{F}$ has been also changed as

$$
\mathscr{L}_{F}=\overline{\mathscr{L}}_{\bar{F}}+\overline{\mathscr{L}}_{\bar{F} \bar{F}} \delta F,
$$

where $\overline{\mathscr{L}}_{\bar{F} \bar{F}}=\partial_{\bar{F}}^{2} \overline{\mathscr{L}}=\partial_{\bar{F}} \overline{\mathscr{L}}_{F}$. Note that $\bar{F}$ and $\overline{\mathscr{L}}_{\bar{F}}$ depend explicitly and implicitly only on $r$, respectively, while, $\mathscr{L}_{F}$ is the function of all coordinates. Now we rewrite the equation of motion (3) in the following form:

$$
\begin{aligned}
\partial_{t}\left(\mathscr{L}_{F} F^{\mu t}\right)+ & \frac{1}{r^{2}} \partial_{r}\left(r^{2} \mathscr{L}_{F} F^{\mu r}\right)+\frac{1}{\sin \theta} \partial_{\theta}\left(\sin \theta \mathscr{L}_{F} F^{\mu \theta}\right) \\
& +\partial_{\phi}\left(\mathscr{L}_{F} F^{\mu \phi}\right)=0
\end{aligned}
$$

For the cases $\mu=t$ and $\mu=r$, above equation have infinite solutions. Therefore, we consider the cases $\mu=\theta$ and $\mu=\phi$ which imply the following equation:

$$
\begin{aligned}
-\frac{\partial^{2} \Psi}{\partial t^{2}}+ & \frac{f}{\overline{\mathscr{L}}_{F}}\left(f \overline{\mathscr{L}}_{F} \Psi^{\prime}\right)^{\prime} \\
& +f \frac{\ell(\ell+1)}{r^{2}}\left(1+\frac{4 Q_{m}^{2} \overline{\mathscr{L}}_{\bar{F} \bar{F}}}{r^{4} \overline{\mathscr{L}}_{\bar{F}}}\right) \Psi=0
\end{aligned}
$$

By introducing the new function (32), and the tortoise coordinate, we arrive again to the wave equation

$$
\left[-\frac{\partial^{2}}{\partial t^{2}}+\frac{\partial^{2}}{\partial x^{2}}-V_{m}(r)\right] \Phi_{m}(r, t)=0,
$$

where the effective potential is now given by the expression

$$
\begin{aligned}
& V_{m}(r)= \\
& f\left[\frac{\ell(\ell+1)}{r^{2}}\left(1+\frac{4 Q_{m}^{2} \overline{\mathscr{L}}_{\bar{F} \bar{F}}}{r^{4} \overline{\mathscr{L}}_{\bar{F}}}\right)-\frac{f \overline{\mathscr{L}}_{\bar{F}}^{\prime 2}-2 \overline{\mathscr{L}}_{\bar{F}}\left(f \overline{\mathscr{L}}_{\bar{F}}^{\prime}\right)^{\prime}}{4 \overline{\mathscr{L}}_{\bar{F}}^{2}}\right] .
\end{aligned}
$$

If we consider linear (Maxwell) electrodynamics, $\overline{\mathscr{L}}_{\bar{F}}=1$, and we recover again the potential (35).

The EM perturbations of the electrically and magnetically charged black holes in the linear EM fields are governed by the same potentials given in (35). On the contrary, the EM perturbations of both electrically (34) and magnetically (43) charged black holes in general relativity coupled to the NED are governed by different potentials and indicate that the electrodynamic nonlinearity must play an important role in behaviour of perturbations (at least for EM perturbations).

\section{QNMS OF MAXWELLIAN REGULAR BLACK HOLE}

As we mentioned already in section III, by changing the values of the parameters $\nu$ and $\mu$ one can construct several different singular and regular black hole solutions. One of the most interesting case of them is the $\nu=1$ case, in which the NED tends to the Maxwell (linear EM) field in the weak field regime as

$$
\mathscr{L}=4 \mu F+O\left(F^{5 / 4}\right) .
$$

Therefore, hereafter, we name $\nu=1$ model as Maxwellian black holes. In this section we study the QNMs of the EM perturbations of these black holes. To see an effect of the NED, we compare the results with those related to the RN black holes.

As other regular black holes or RN black hole spacetimes, the Maxwellian regular black holes also have two horizons: inner $r_{-} \geq 0$ and outer $r_{+} \leq 2 M$, for $q<q_{e x t}$, one horizon $r_{-}=r_{+}=r_{e x t}$ for $q=q_{e x t}$, or no horizon (naked singularity for the RN spacetime) for $q>q_{\text {ext }}-$ see Fig. 11. For the RN black hole: $q_{\text {ext }}=M$ and $r_{\text {ext }}=M$. For the Maxwellian regular black hole with $\mu=3: q_{\text {ext }} \approx 0.2963 M$ and $r_{\text {ext }} \approx$ $0.5926 M, \mu=4: q_{\text {ext }} \approx 0.2109 M$ and $r_{\text {ext }} \approx 0.6328 M$, $\mu=5: q_{\text {ext }} \approx 0.1638 M$ and $r_{\text {ext }} \approx 0.6554 M$. F Note that we are using the same notation $q$ for the magnetic (or electric) charge of the RN black hole and magnetic charge of the Maxwellian regular black holes.

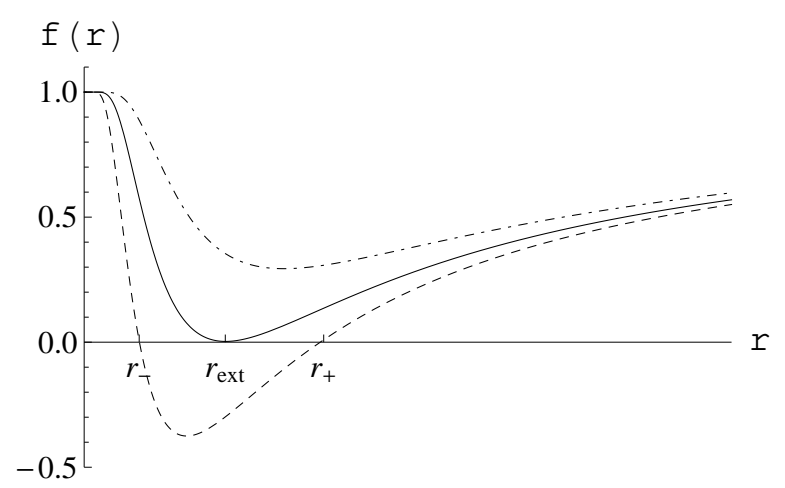

FIG. 1. Radial dependence of the metric function $f(r)$ given by (19.). Here we set $M=1$. Dashed curve represents black hole, solid curve represents extreme black hole, and dotdashed curve represents no-horizon spacetimes.

One can notice that the range of possible values of magnetic charge parameter of the Maxwellian regular black holes is tighter than the one of the RN black hole. Therefore, in order to simplify the comparison, we normalize their values by introducing the new parameter $Q \equiv q / q_{\text {ext }}$. To study the QNMs one should analyze the effective potentials of the Maxwellian and RN black holes. Due to the cumbersome length of the effective potential (43) for the whole range of parameter $\mu$ of the Maxwellian regular black holes (22), we report here only for the minimum value of the parameter $\mu$ for the black hole to be regular, i.e., $\mu=3$ as

$$
\begin{aligned}
& V=f\left[\frac{\ell(\ell+1)(2 r-3 q)}{2 r^{2}(q+r)}\right. \\
& \left.\frac{5 q\left(3 r^{3}(4 M-3 q)-q r^{2}(14 M+3 q)+3 q^{4}+5 q^{3} r-4 r^{4}\right)}{4 r^{2}(q+r)^{5}}\right] .
\end{aligned}
$$

In Fig. 目 we compare the effective potentials of the RN (35) and regular Maxwellian black holes (45) for the same normalized charge parameters. One can see from Fig. 2 that outside

\footnotetext{
5 These values can be easily obtained by solving the equations $f=0$ and $f^{\prime}=0$, simultaneously.
} 


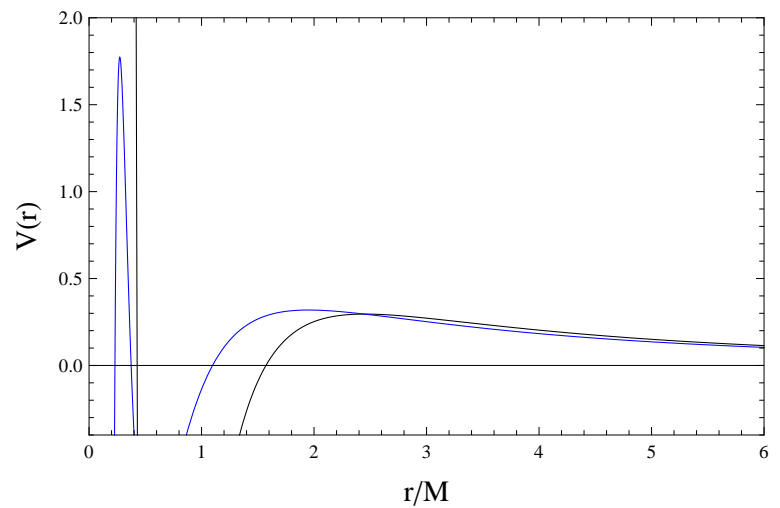

FIG. 2. Radial dependence of effective potentials of the EM perturbations of black holes in nonlinear (Maxwellian black hole with $\mu=3$, blue curve) and linear (RN, black curve) electrodynamics. Where we set the values of the charge equal $Q=0.8 M$.

the event horizon of the black holes both RN and Maxwellian regular black holes have very similar potential barriers which tend to zero at infinity. However, unlike the case in the RN black hole, inside the event horizon of the Maxwellian black holes, there is another very narrow potential barrier, located between the inner horizon $r_{-}$and another zero of the effective potential $r_{0}$, which depends on $\ell$ and $q / M$. In Fig. 3 dependence of the location of $r_{0}$ on $q$ for several values of $\ell$ is presented.

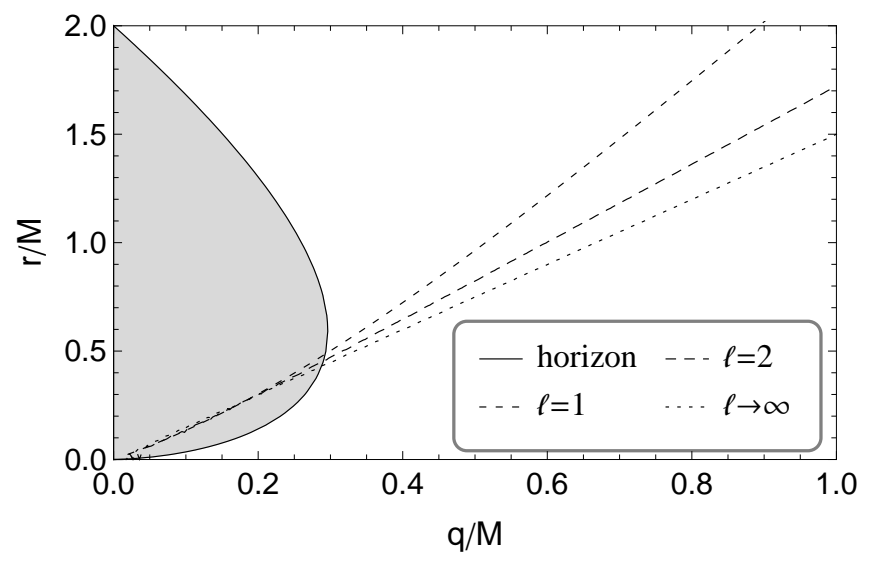

FIG. 3. Location of $r_{0}$ depending on the charge parameter $q$ for several values of multipole number $\ell$. The shaded and white regions represent the black hole and no horizon spacetimes.

As one sees from Fig. 3, the location of $r_{0}$ is almost independent of multipole number $\ell$, but it is almost linearly dependent on the charge $q$. Figs. 目 and 3 show that in the Maxwellian black holes in the region $r \in\left[r_{-}, r_{0}\right] \cup\left[r_{+},+\infty\right)$ there are two potential barriers. Both of them increase with increasing multipole number $\ell$. However, their dependence on the charge parameter are different: with increasing charge parameter, the inner barrier dramatically decreases, while the outer one increases. Despite these discussion, one must note that according to the classical physics, there is nothing com- ing out from black hole. Therefore, we do not consider the inner potential barrier. Another really interesting property of the Maxwellian regular black hole is that even in the no horizon spacetimes, the effective potential keeps its barrier form outside $r_{0}$, i.e., $r \in\left[r_{0},+\infty\right)$.

\section{A. The temporal evolution of perturbations}

We study the evolution of the EM perturbations by using a characteristic integration method [34. 35] that involves the light-cone variables: retarded $d u \equiv d t-d x$ and advanced $d v \equiv d t+d x$ time coordinates, with initial data specified on the two null surfaces $u=u_{0}$ and $v=v_{0}$. The wave equation (42) then takes the form

$$
-4 \frac{\partial^{2} \Phi}{\partial u \partial v}=V(r(u, v)) \Phi .
$$

This equation is solved numerically. The $(u, v)$ space is divided into finite grid with constant $\Delta$ separating neighboring points of the grid. The numerical scheme used to solve this equation reads

$$
\Phi_{N}=\left(\Phi_{W}+\Phi_{E}\right) \frac{16-\Delta^{2} V_{S}}{16+\Delta^{2} V_{S}}-\Phi_{S}
$$

where the indices $N, W, E$, and $S$ refer to grid-points $N \equiv(u, v), W \equiv(u-\Delta, v), E \equiv(u, v-\Delta)$, and $S \equiv(u-\Delta, v-\Delta)$. In our simulations the initial perturbation is Gaussian function centered around the point $x_{c}$ (in tortoise coordinates) and it takes the form

$$
\begin{aligned}
\Phi(t=0, x) & =A \exp \left(-\left(x-x_{c}\right)^{2} / \sigma^{2}\right) \\
& =A \exp \left(-\left(v-v_{c}\right)^{2} / \sigma^{2}\right)
\end{aligned}
$$

since

$$
t=0=\frac{1}{2}(u+v) \Rightarrow u=-v
$$

and therefore

$$
x=\frac{1}{2}(v-u)=v
$$

for $t=0$. At the center of the body, $x=0$, we initially put the boundary condition $\Phi(u, v)=0$ which is considered along the line $u=v$ since for

$$
x=0=\frac{1}{2}(v-u) \Rightarrow u=v .
$$

Our tortoise coordinate $x$ is determined from the formula

$$
x=\int_{0}^{r} \frac{1}{f\left(r^{\prime}\right)} \mathrm{d} r^{\prime}
$$

which implies that $x \geq 0$ for $r \geq 0$. We are therefore interested only in the region where $v \geq u$. In the integration loop the coordinates $u$ and $v$ are determined by formulas

$$
u=i_{u} \Delta, \quad \text { where } i_{u}=\{1,2, \ldots, N\}
$$




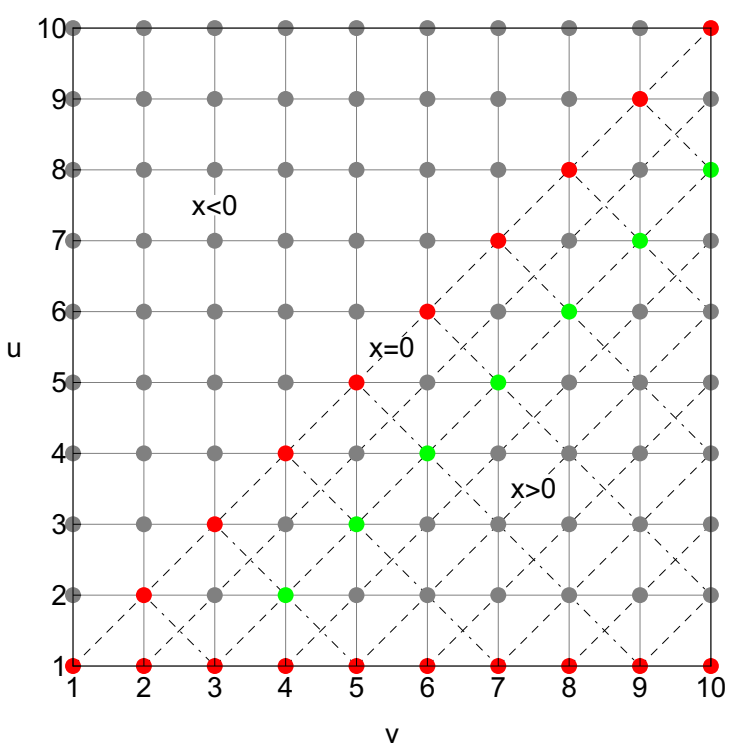

FIG. 4. The discretized numerical grid $(u, v)$. The red dots represent initial values (horizontal) and boundary values (diagonal). The dashed lines correspond to fixed coordinate $x$ while dot-dashed lines correspond to fixed coordinate $t$. The green dots here represent the solution at a chosen, fixed coordinate $x$.

and

$$
v=i_{v} \Delta, \quad \text { where } i_{v}=\left\{i_{u}+1, i_{u}+2, \ldots, N\right\} .
$$

We illustrate the temporal evolution of the EM perturbations of the NED Maxwellian regular black holes and the RN black holes in Fig. 5. One can see from Fig. 5 that the main difference of the evolution of the EM perturbations in the black holes in the linear and nonlinear electrodynamics is that an increase in the value of the charge parameter of the NED Maxwellian regular black holes prolongs perturbations, while in the linear electrodynamics, it shortens the life of the EM perturbations. Note that in small and intermediate values of the charge parameters of the Maxwellian and RN black holes evolution of the EM perturbations are almost the same.

Moreover, time domain profiles of the EM perturbations of the Maxwellian regular black holes show that they are stable against EM perturbations.

\section{B. QN frequencies}

In this subsection we calculate QN frequencies by considering the EM perturbations to be harmonically time dependent as

$$
\Phi(r, t)=\psi(r) e^{-i \omega t},
$$

with $\omega=\omega_{r}+i \omega_{i}$ being the QN frequency, $\omega_{r}$ represents frequency of the oscillations, while $\omega_{i}<0\left(\omega_{i}>0\right)$ represents damping (growing) of these oscillations. Then, the master equation (42) takes the new form

$$
\left(\frac{\partial^{2}}{\partial x^{2}}+\omega^{2}-V\right) \psi=0 \text {. }
$$

Since we are going to study the effect of the nonlinearity of the electrodynamic field, here $V$ is given by the potentials (43) and (35). Since these potentials vanish at the horizon $(x=$ $-\infty)$ and tend to zero at infinity $(x=+\infty)$, we choose the boundary condition such that at horizon (infinity) the wave is purely incoming (outgoing) as

$$
\psi \sim e^{\mp i \omega t}, \quad x \rightarrow \mp \infty .
$$

Solving the Eq. (56) with the effective potential (45) and boundary conditions (57) analytically is impossible. Therefore, to solve this equation, we use the well-known semianalytical method, the sixth order WKB method [36, 37]. Calculations show that the QN frequencies of the EM perturbations of the regular Maxwellian black holes with $\mu=3$ are almost the same as the ones related to the RN black holes. Therefore, we do not report all the numerical results; in Fig. 6 in order to ease comparison, we present some of these results. Moreover, in Fig. 7) we present some nonfundamental QNMs of the axial EM perturbation of the regular Maxwellian black holes.

\section{EIKONAL QNMS}

In the paper [38] it has been shown that in the general relativity framework, the QNMs of any stationary, spherically symmetric and asymptotically flat black holes in any dimensions are determined in the eikonal (large multipole number) regime by the parameters of the circular null geodesics, i.e., the real part of the QN frequencies is determined by angular velocity of the unstable null geodesics, $\Omega_{c}$, while the imaginary part of the QN frequencies is determined by the instability timescale of the orbit, so called Lyapunov exponent, $\lambda$. The frequency is thus given by the relation 38.

$$
\omega=\Omega_{c} \ell-i\left(n+\frac{1}{2}\right)|\lambda|,
$$

where $\Omega_{c}$ and $\lambda$ for the spacetime metric (5) are given by the following expressions

$$
\begin{aligned}
& \Omega_{c}=\sqrt{\frac{f_{c}}{r_{c}^{2}}}, \\
& \lambda=\sqrt{-\left.\frac{r_{c}^{2}}{2 f_{c}}\left(\frac{d^{2}}{d x^{2}} \frac{f}{r^{2}}\right)\right|_{r=r_{c}}},
\end{aligned}
$$

where $x$ is the tortoise coordinate, $r_{c}$ is radius of the unstable null circular orbit which is determined by the solution of equation $r_{c} f_{c}^{\prime}-2 f_{c}=0$. However, (58) is not universal feature of all stationary, spherically symmetric and asymptotically flat black holes in any dimensions, as it has been shown in [39, 40] that these phenomena are violated in the Einstein-Lovelock theory. Formally, the same conclusion that the eikonal QNMs of the EM perturbations are not related to the circular null geodesics, holds for the metric of the regular black holes considered in this paper.

However, in our case the situation is slightly different than in the Einstein-Lovelock gravity. Let us write the effective 

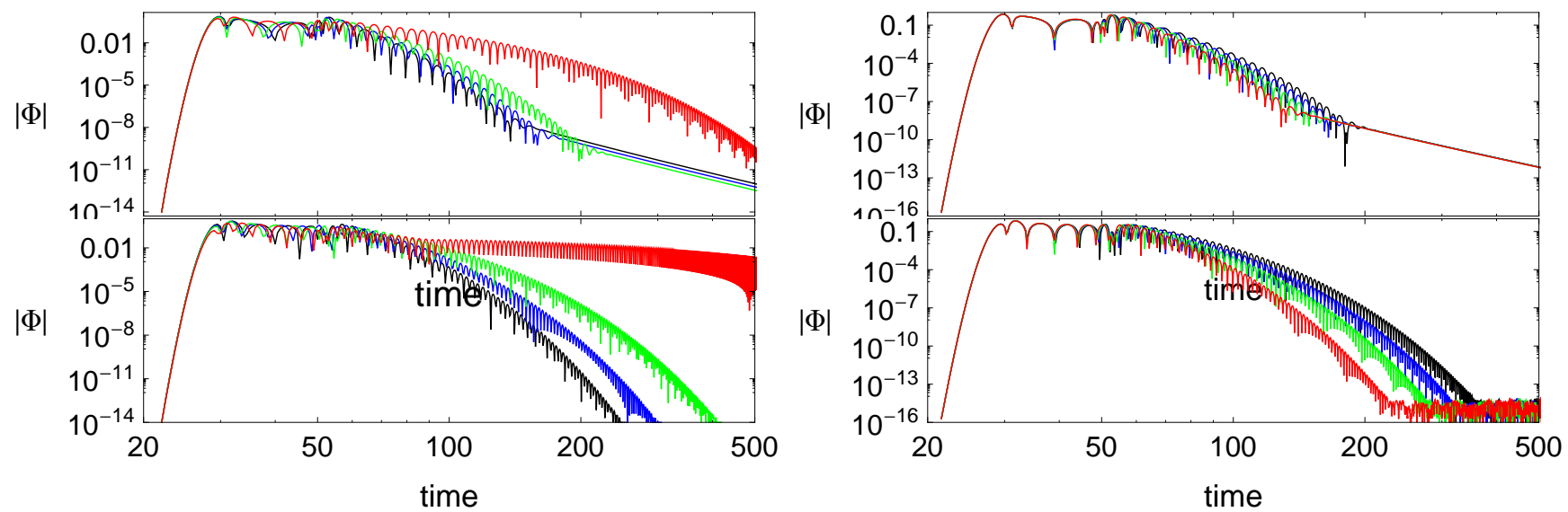

FIG. 5. Temporal evolution of $\ell=2$ (top panel) and $\ell=4$ (bottom panel) fundamental modes of the EM perturbations of the Maxwellian regular (left panel) and the RN (right panel) black holes for the values $Q=0.2$ (black), $Q=0.6$ (blue), $Q=0.8$ (green), and $Q=0.998$ (red).

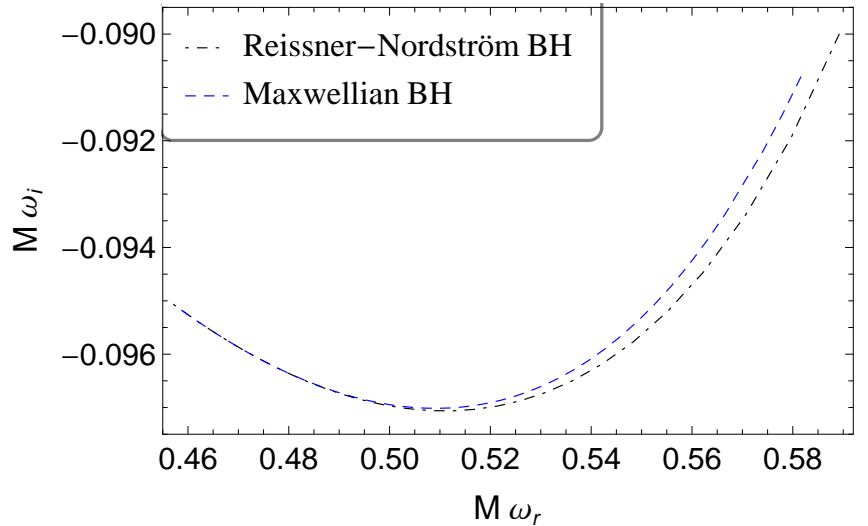

FIG. 6. $\ell=2, n=0 \mathrm{QN}$ frequencies of the EM perturbations of the Maxwellian and RN black holes with the normalized charge, $Q \in[0,1]$, where $Q=0$ is located at the junction of the curves which corresponds to the Schwarzschild black hole.

potential of the EM perturbation (43) for the large multipole number regime as

$$
V=\ell^{2}\left[\frac{f}{r^{2}}\left(1+\frac{4 Q_{m}^{2} \overline{\mathscr{L}}_{\bar{F} \bar{F}}}{r^{4} \overline{\mathscr{L}}_{\bar{F}}}\right)+O\left(\frac{1}{\ell}\right)\right],
$$

It is obvious from the potential (61) that to find the eikonal QNMs, expressions (59) and (60) do not work. The potential 61) corresponds to the one of the photon motion (NOT NULL GEODESICS) around NED Maxwellian black holes. It is well know that in the NED, photon does not follow the null geodesics of original metric, instead, it follows the null geodesics of the effective optical metric [3, 18,23]. The effective metric can be constructed as

$$
g_{\text {eff }}^{\mu \nu}=\overline{\mathscr{L}}_{\bar{F}} g^{\mu \nu}-4 \overline{\mathscr{L}}_{\bar{F} \bar{F}} \bar{F}_{\alpha}^{\mu} \bar{F}^{\alpha \nu} .
$$

For the magnetically charged Maxwellian black hole with the line element (5), covariant components of the effective metric

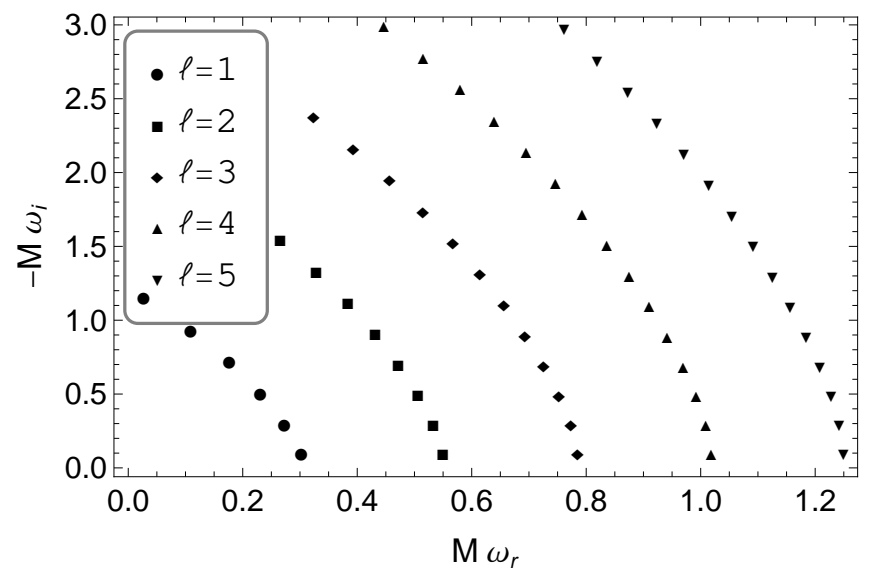

FIG. 7. Nonfundamental QN frequencies of the EM perturbation of Maxwellian regular black hole with $Q=0.8$ for several values of the multipole number $\ell$. The overtone number, $n$, increases as $n=0,1,2, \ldots$ from bottom to top.

tensor can be written as the conformal transformation of the covariant metric tensor $\left(g_{\mu \nu}^{e f f}=\Omega^{2} g_{\mu \nu}\right)$ as

$g_{\mu \nu}^{e f f}=\left(\overline{\mathscr{L}}_{\bar{F}}+\frac{4 Q_{m}^{2} \overline{\mathscr{L}}_{\bar{F} \bar{F}}}{r^{4}}\right)^{-1} \operatorname{diag}\left\{-g, \frac{1}{h}, r^{2}, r^{2} \sin ^{2} \theta\right\}$.

with

$$
g=f\left(1+\frac{4 Q_{m}^{2} \overline{\mathscr{L}}_{\bar{F} \bar{F}}}{r^{4} \overline{\mathscr{L}}_{\bar{F}}}\right), \quad h=\frac{f}{\left(1+\frac{4 Q_{m}^{2} \overline{\mathscr{L}}_{\bar{F} \bar{F}}}{r^{4} \overline{\mathscr{L}}_{\bar{F}}}\right)}
$$

It has been shown that the conformal factor $\Omega^{2}$ plays no role in the EM perturbations [41] and null geodesics [42]. Now 
one can find the parameters of circular photon orbit as

$$
\begin{aligned}
& \Omega_{c}=\sqrt{\frac{g_{c}}{r_{c}^{2}}}, \\
& \lambda=\sqrt{\frac{h_{c}}{2 r_{c}^{2}}\left(2 g_{c}-r_{c}^{2} g_{c}^{\prime \prime}\right)},
\end{aligned}
$$

where $r_{c}$ is determined by equation $r_{c} g_{c}^{\prime}-2 g_{c}=0$. In Fig. 8 radii of the circular unstable null geodesics of the RN and Maxwellian black holes are depicted. Numerical calculations

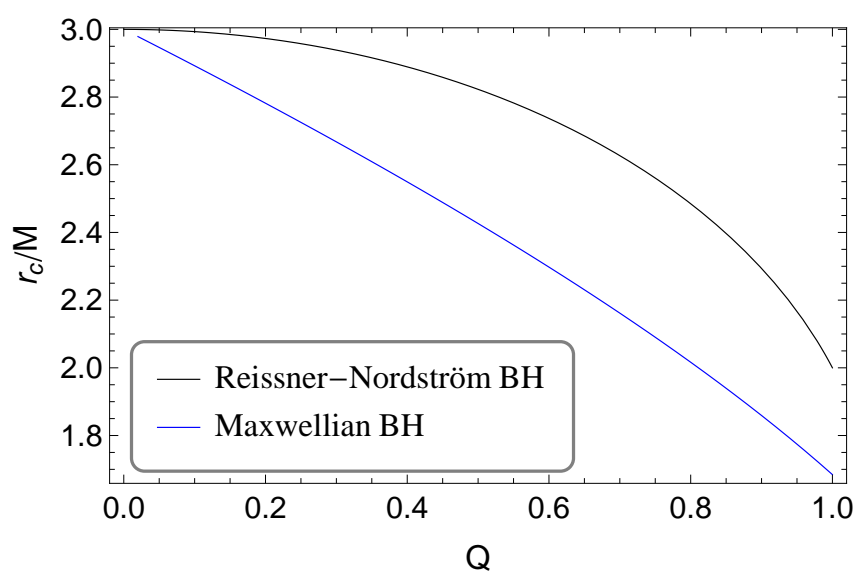

FIG. 8. Dependence of radius of circular unstable null geodesics on the normalized charge parameter of RN (black) and Maxwellian regular (blue) black holes.

show that formula of the eikonal QN frequencies (58) works finely in the EM perturbations of the NED black holes only if instead of the parameters of the circular null geodesics, the parameters of the circular photon orbit are used. In Fig. 9 the angular velocity of the circular unstable photon orbit (65) and the Lyapunov exponent (66) of the RN and Maxwellian regular black holes are presented.

One can see from Fig. 9 that in the large multipole numbers limit, the EM perturbation of the Maxwellian regular black hole propagates the QNMs with bigger real frequencies than the $\mathrm{RN}$ one with almost the same decaying rates.

\section{CONCLUSION}

In the present paper we demonstrated the formalism to construct the electrically and magnetically charged (singular and regular) black hole solutions in general relativity coupled to the NED. For our special interest, we constructed the family of new singular NED black hole solutions which tends to the linear (Maxwell) electrodynamics in the weak field limit, based on the Lagrangian density supposed in 肺. We showed that these solutions are singular at $r=0$ and unlike the other standard singular solutions, these solutions are convertible to the regular ones by the special condition: $M=q^{3} / \alpha$. As usual regular black hole spacetimes, these Maxwellian regular black hole spacetimes also represent black hole, extremal black hole, and no horizon spacetimes depending on the values of the gravitational mass and NED parameters.

The main part of this paper is dedicated to the study of the axial EM perturbations of the general NED black hole solutions considering the EM perturbations that do not alter the spacetime geometry. We showed that the EM perturbations of the NED black holes give different potentials and, consequently, different results for the QN frequencies, as compared to those related to the RN black holes in the standard electrovacuum theory. It is well known that the EM perturbations of the electrically and magnetically charged black holes in linear electrodynamics (RN) are isospectral, i.e., they have the same effective potentials and QN frequencies, however, in the case of the NED black holes, electrically and magnetically charged black holes have different potentials and different QNM spectra. As a special case, we calculated QNMs of the magnetically charged Maxwellian regular black hole with $\mu=3$ and compared them with the ones of the $\mathrm{RN}$ black holes by normalizing the charge parameter as $Q=q / q_{\text {ext }}$ where $Q \in[0,1]$. The analysis of the time domain profile and the QNM frequencies show that the Maxwellian regular black holes are stable against EM perturbations.

In the paper [38] it was stated that in the eikonal (high energy or large multipole number) limit QNMs related with the unstable circular null geodesics. In this paper we showed by the EM perturbations of the NED black holes that this claim is correct in the standard linear electrodynamics, however, it does not work in the NED, since in the NED photon does not follow the null geodesics, instead it follows the null geodesics of an effective metric. We claim that in the eikonal regime, the QNMs of NED black holes are determined by the unstable circular photon orbits determined by the effective geometry.

\section{ACKNOWLEDGEMENTS}

B.T. would like to thank Roman Konoplya for reading the previous version of manuscript and providing fruitful discussions and comments. B.T., Z.S., and J.S. would like to acknowledge the institutional support of the Faculty of Philosophy and Science of the Silesian University in Opava, the internal student grant of the Silesian University (Grant No. SGS/14/2016) and the Albert Einstein Centre for Gravitation and Astrophysics under the Czech Science Foundation (Grant No. 14-37086G). The researches of B.A. and B.T. are partially supported by Grants No. VA-FA-F-2-008 and No. YFA-Ftech-2018-8 of the Uzbekistan Ministry for Innovation Development, by the Abdus Salam International Centre for Theoretical Physics through Grant No. OEA-NT-01. B.T. and B.A. would like to acknowledge Nazarbayev University, Astana, Kazakhstan for the warm hospitality through support from ORAU grant SST 2015021. 

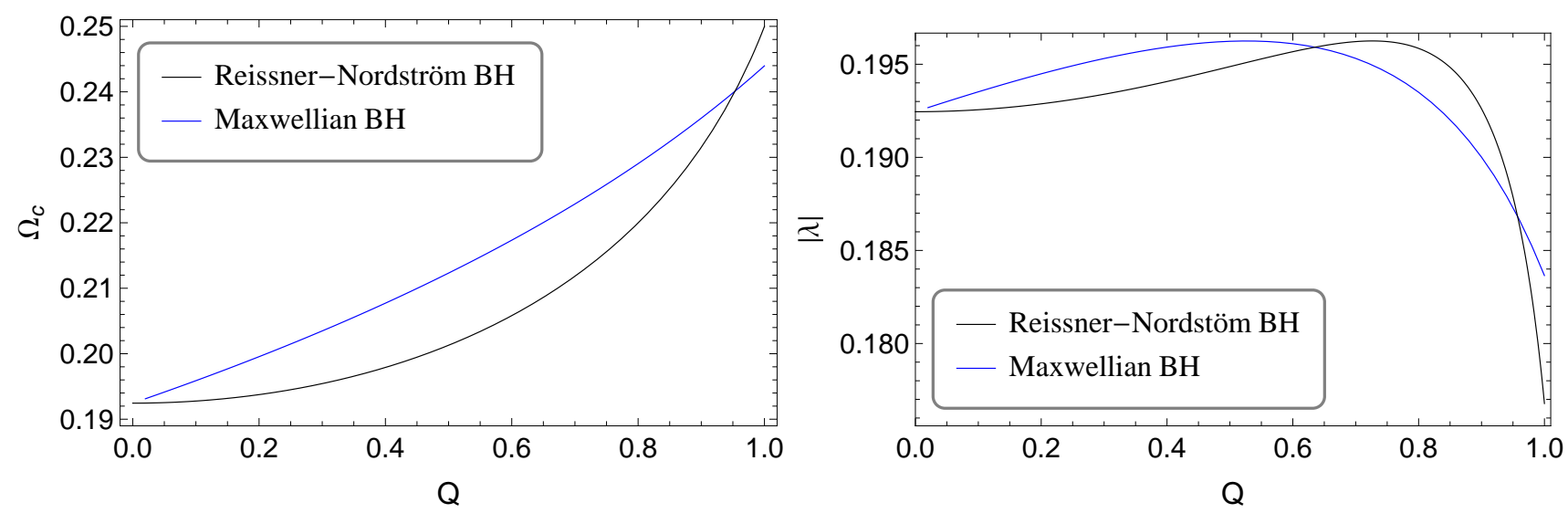

FIG. 9. Dependence of angular velocity and lyapunov exponent of circular unstable null geodesics on the normalized charge parameter of RN (black) and Maxwellian regular (blue) black holes.

[1] E. Ayón-Beato and A. García, Phys. Rev. Lett. 80, 5056 (1998) gr-qc/9911046.

[2] E. Ayón-Beato and A. García, Phys. Lett. B 493, 149 (2000), gr-qc/0009077

[3] K. A. Bronnikov, Phys. Rev. D 63, 044005 (2001)] grqc/0006014.

[4] I. Dymnikova, Classical and Quantum Gravity 21, 4417 (2004), gr-qc/0407072.

[5] Z.-Y. Fan and X. Wang, Phys. Rev. D 94, 124027 (2016), arXiv: 1610.02636 [gr-gc]

[6] K. A. Bronnikov, Phys. Rev. D 96, 128501 (2017), arXiv:1712.04342 [gr-qc].

[7] J. E. McClintock, R. Shafee, R. Narayan, R. A. Remillard, S. W. Davis, and L.-X. Li, Astrophys. J. 652, 518 (2006), astro$\mathrm{ph} / 0606076$.

[8] B. P. Abbott, R. Abbott, T. D. Abbott, M. R. Abernathy, F. Acernese, K. Ackley, C. Adams, T. Adams, P. Addesso, R. X. Adhikari, and et al., Phys. Rev. Lett. 116, 061102 (2016), arXiv: 1602.03837 [gr-qc].

[9] E. T. Newman and A. I. Janis, J. Math. Phys. 6, 915 (1965).

[10] C. Bambi and L. Modesto, Phys. Lett. B 721, 329 (2013), arXiv:1302.6075 [gr-qc]

[11] B. Toshmatov, B. Ahmedov, A. Abdujabbarov, and Z. Stuchlík, Phys. Rev. D 89, 104017 (2014), arXiv: 1404.6443 [gr-qc].

[12] M. Azreg-Aïnou, Phys. Rev. D 90, 064041 (2014), arXiv: 1405.2569 [gr-qc]

[13] B. Toshmatov, Z. Stuchlík, and B. Ahmedov, Phys. Rev. D 95, 084037 (2017), arXiv:1704.07300 [gr-qc].

[14] M. Gürses and F. Gürsey, I. Math. Phys. 16, 2385 (1975).

[15] I. Dymnikova and E. Galaktionov, Classical and Quantum Gravity 32, 165015 (2015), arXiv:1510.01353 [gr-qc].

[16] M. E. Rodrigues and E. L. B. Junior, Phys. Rev. D 96, 128502 (2017), arXiv:1712.03592 [gr-qc]

[17] J. C. S. Neves and A. Saa, Phys. Lett. B 734, 44 (2014), arXiv: 1402.2694 [gr-qc]

[18] M. Novello, V. A. De Lorenci, J. M. Salim, and R. Klippert, Phys. Rev. D 61, 045001 (2000), gr-qc/9911085.

[19] M. Novello, J. M. Salim, V. A. De Lorenci, and E. Elbaz, Phys. Rev. D 63, 103516 (2001).
[20] Y. N. Obukhov and G. F. Rubilar, Phys. Rev. D 66, 024042 (2002), gr-qc/0204028.

[21] N. Bretón and L. A. López, Phys. Rev. D 94, 104008 (2016), arXiv: 1607.02476 [gr-qc].

[22] E. G. de Oliveira Costa and S. E. Perez Bergliaffa, Classical and Quantum Gravity 26, 135015 (2009), arXiv:0905.3673 [gr-qc]

[23] Z. Stuchlík and J. Schee, Int. J. Mod. Phys. D 24, 1550020-289 (2015), arXiv:1501.00015 [astro-ph.HE].

[24] N. Bretón, Phys. Rev. D 72, 044015 (2005), hep-th/0502217.

[25] C. Moreno and O. Sarbach, Phys. Rev. D 67, 024028 (2003), gr-qc/0208090

[26] N. Bretón and S. E. P. Bergliaffa, Annals of Physics 354, 440 (2015).

[27] H. Nomura and T. Tamaki, Phys. Rev. D 71, 124033 (2005), hep-th/0504059.

[28] S. Fernando and J. Correa, Phys. Rev. D 86, 064039 (2012), arXiv:1208.5442 [gr-qc].

[29] A. Flachi and J. P. S. Lemos, Phys. Rev. D 87, 024034 (2013), arXiv:1211.6212 [gr-qc]

[30] J. Li, H. Ma, and K. Lin, Phys. Rev. D 88, 064001 (2013), arXiv: 1308.6499 [gr-qc]

[31] B. Toshmatov, A. Abdujabbarov, Z. Stuchlík, and B. Ahmedov, Phys. Rev. D 91, 083008 (2015) arXiv:1503.05737 [gr-qc],

[32] J. Li, K. Lin, and N. Yang, Eur. Phys. J. C 75, 131 (2015), arXiv: 1409.5988 [gr-qc].

[33] E. Chaverra, J. C. Degollado, C. Moreno, and O. Sarbach, Phys. Rev. D 93, 123013 (2016), arXiv: 1605.04003 [gr-qc],

[34] C. Gundlach, R. H. Price, and J. Pullin, Phys. Rev. D 49, 883 (1994), gr-qc/9307009.

[35] C. B. M. H. Chirenti and L. Rezzolla, Classical and Quantum Gravity 24, 4191 (2007), arXiv:0706.1513 [gr-qc).

[36] R. A. Konoplya, Phys. Rev. D 68, 024018 (2003), grqc/0303052.

[37] B. Toshmatov, Z. Stuchlík, J. Schee, and B. Ahmedov, Phys. Rev. D 93, 124017 (2016), arXiv:1605.02058 [gr-qc].

[38] V. Cardoso, A. S. Miranda, E. Berti, H. Witek, and V. T. Zanchin, Phys. Rev. D 79, 064016 (2009), arXiv:0812.1806 [hep-th].

[39] R. A. Konoplya and Z. Stuchlík, Phys. Lett. B 771, 597 (2017), arXiv:1705.05928 [gr-qc]. 
[40] R. A. Konoplya and A. Zhidenko, J. Cosmo . Astropart. Phys. 5, 050 (2017), arXiv:1705.01656 [hep-th].

[41] B. Toshmatov, C. Bambi, B. Ahmedov, ł. Stuchlík, and J. Schee, Phys. Rev. D 96, 064028 (2017), arXiv:1705.03654 [gr-qc].

[42] C. Banłbi, L. Modesto, and L. Rachwał, J. Cosmol. Astropart. Phys. \$, 003 (2017), arXiv:1611.00865 [gr-qc]. 